\title{
A New Communication Protocol for Drones Cooperative Network: 5G Site Survey Case Study
}

\author{
Youssef Shawky Othman ${ }^{1}$, Dr. Mohamed Helmy \\ Megahed $^{2}$, Prof. Dr. Mohamed Abo Rezka ${ }^{3}$ \\ Computer Science \\ Arab Academy for Science and Technology and Maritime \\ Transport, Cairo, Egypt
}

\author{
Prof. Dr. Fathy Ahmed Elsayed Amer ${ }^{4}$ \\ Prof. Dr. Comp. Sci. Dep \\ College of Info. Sys.,Comp. Sci., Oct. 6, Univ \\ Cairo, Egypt
}

\begin{abstract}
Directing the antennas of the 5th generation mobile network optimally became a hard and tedious process due to the abundance of the antennas that the 5th generation networks relay on. Also, due to the traditional way of measuring the signal strength of the 5th generation networks. The process that could take weeks of working until it is done. So, the solution is to make an automated process to measure signal strength and to direct antennas using "Drones" rather than human power. This way will ease the process of directing antennas in a quite shorter amount of time which will be some hours instead of weeks. Plus the low cost and the high accuracy achieved. So, a cooperative network between "Drones" and a new communication protocol to support that network will be designed. "Drones" will communicate with each others and with antennas through exchanging messages by "MQTT cloud" using new designed communication protocol. "Raspberry pi" platform will be used as a server to control the direction of antennas. "Drones" will carry a "4G mobile" and a "Raspberry pi" with a "Building Identification System (BIS)" setuped on it. The "(BIS)" gives every building a number and will recognize the entrance of buildings to measure signal strength at every floor. Then, performance analysis metrics (Throughput) will be measured. OMNeT++ "will be used for simulation", and "Raspberry Pi" platform will be used to implement the system and measure the performance of the new Communication Protocol.
\end{abstract}

Keywords-Drones; communication protocol; Message Queuing Telemetry Transport (MQTT); cooperative network

\section{INTRODUCTION}

The fifth generation of mobile communication systems aims to provide a ubiquitous mobile service with better quality. Automobiles, public transportation, medical care, energy, public safety, agriculture, entertainment, manufacturing, and other vertical industrial applications are expected to benefit from this technology. User density, traffic volume, and data rate are all expected to increase dramatically. In the coming decade, innovative solutions to the needs of both smartphone users and vertical industries are needed.

There are many challenges to realize 5G networks, i.e., high system capacity (1000× capacity per km2), high data rates (targeting 1 Gbps per user everywhere and 100× user throughput increase) and massive connectivity (100× connected users) [1]. The advent of fifth generation (5G) technology will accelerate the growth of V2V communications. A recent report from Juniper Research predicted that as 5G technology provides lower latency and high range, automotive Original Equipment Manufacturer (OEMs) will use it as the main technology for $\mathrm{V} 2 \mathrm{~V}$ communications over other technologies [2].

The traditional way that is used now in The fifth generation (5G) of mobile communication system to measure the coverage cell phone network signal strength in cellular base stations is through the human power using smartphones, where specialists go to the antennas above the base stations to direct it. The different teams of specialists are distributed over the whole places and buildings that is under the coverage of the base station. Then they take the measure of signal strength via a mobile application like "Network cell info lite" then they communicate with the specialists on the base station and deliver the measures taken to them so that they can direct the antennas according to what they received. This process that repeated over and over again in an attempt to get the optimal direction of the antennas. But finally it doesn't get achieved for several reasons.. First is the high cost. Second is the high consumption of time "that could take days and sometimes a few weeks" and third is the lack of accuracy from the human power. Besides some changes that may happen to the coverage places in terms of the buildings and its height or the continuous variation in the number of users of the network. Which leads us to repeat the whole process many times in small period of times. Which is a big obstacle to telecommunication companies.

With advances in technology and commercially available vehicles, the interest is shifting toward collaborative drones. cooperation necessitates effective communication as well as a sound strategy for avoiding obstacles and drone collisions. Considering that drones must swap positions in order to maintain the swarm formation, cover a large area, and avoid colliding [3].

Several projects searched into the challenges of designing UAV systems for various applications. The general design principles of a multi-UAV system in civil applications are an open issue and are still being researched [4].

According to all the mentioned before, it becomes necessary to think \& research to find an alternative way (using "Drones" to direct antennas) instead of the traditional way that has lots of disadvantages like the high cost, the high consumption of time and the lack of accuracy. 
These disadvantages that led to the weak signal or no signal at all in mobile networks due to the poor distribution of antennas and not directing them optimally that finally make the mobile users suffer when they use the network specifically in higher floors in skyscrapers. So to avoid all these problems, the proposal is:

Replacing the human power with "Drones" by creating a cooperative network between each others. As "Drones" will be sent to the buildings and places that are under the coverage to take the measures of the signal strength. And by the connection between the "Drones" and the "Server" placed on the base station, the measures will be sent to it through MQTT Cloud (or off load when facing no connection situation). Then, by the "Servo motors" the antennas will be directed to the optimal angle.

As a result the solution presented will lead us to avoid all the disadvantages mentioned before there for there is no chance to face any high cost caused by repeating the process in the traditional way.

Contributions :

- Building Cooperative Network Between Drones.

- Building New Communication Protocol To support Cooperative Network Between Drones

- Design and implementation for mobile application on MQTT cloud

- Make a Hardware Implementation using raspberry pi for receiving the data from drones to do tilting of antenna.

Outlines of paper:

Section II presents "Related work" (Cooperative network of drones, 5G, ordinary site survey, image processing, mobile application, MQTT, hardware implementation). Section III presents "Overall Proposed model". Section IV presents "Drones Cooperative network". Section V presents "Proposed new communication protocol". Section VI presents "Mobile application". Section VII presents "Building Identification System (BIS) and Directing Antenna System (DAS)". Section VIII presents "Simulation results and Performance analysis". Section IX presents "Hardware implementation". Section X presents "Comparison with other works". Section XI presents "Conclusion and future work".

\section{RELATED WORK}

The rapid growth of Unmanned Aerial Vehicles (UAVs) for various applications such as package delivery, inspection, defence, and disaster relief has opened up a plethora of commercial business opportunities. For successful use of UAVs for various applications, it is critical that the vehicles be configured to operate autonomously and cooperatively.

In this section, related work will be reviewed. Also the Cooperative network of drones, 5G, ordinary site survey, image processing, mobile application, MQTT, and hardware implementation of Cooperative Network of Drones will be reviewed.
In [3] Bekhti, et al. presented The application of a swarm of drones to the intrusion detection and tracking problem. They devised an algorithm to keep the drones in a team formation in order to address the issue of coverage and collisions. Omnet simulator was used to test the algorithm in various scenarios.

In [5] Gu, et al. they proposed a cooperative network platform and system architecture of multi-UAV surveillance, they discussed the establishment of suitable algorithms based on machine learning.

In [4] presented by Yanmaz, et al. they proposed drones are being utilized in monitoring, transport, safety and disaster management, other domains. they described a high-level architecture for the design of a collaborative aerial system consisting of drones with on-board sensors and embedded processing, coordination, and networking capabilities.

In [6] Dong, et al. they studied optimizing the deployment density of Drone Small Cells (DSCs) to achieve the maximum coverage performance, they propose an algorithm to get the optimal deployment density with low complexity. they conducted both field experiments and Matlab simulations to verify the correctness of theoretical analysis.

In [1] Nirwan and Liang they investigated the problem of Placement and communications in the Drone-mounted basestations (DBS) to provide ubiquitous connections and high spectrum efficiency.

In [7] Chakrabarty, et al. they simulated a complete urban operations in a high fidelity simulation environment. they designed a V2V communication protocol and all the vehicles "Drones" participating communicate over this system.

In [8] Manasa, et al. they discussed a new approach to the wireless communication between Ground Control Station (GCS) to Micro Air Vehicle (MAV) and vice versa.

In [9] Iranmanesh, et al. they discussed congestion problem in cellular networks through the assistance of parcel delivery drones. To this end, they proposed a novel algorithm, called CARLO.

In [10] $\mathrm{Ma}$, et al. they utilized drones as air routers to establish a LAN, to collect information from pipeline networks and transmit it to pipeline inspectors. to achieve optimal drone deployment. A two-phase evolution optimal 3-D drone layout algorithm was proposed to deploy drones.

In [11] Buksz, et al. they presented an approach for autonomous radio base station inspection using cooperative drones based on Intent-driven Strategic Tactical Planning (ISTP).

In [12] Lee. He proposed a cooperative drones positioning measuring in internet-of-drones in order to obtain high accuracy positions for both egodrones and obstacles.

In [13] Bartolini, et al. They work addressed the problem of assigning location based tasks to a fleet of drones in an emergency critical scenario, to ensure early inspection of target locations.

In [14] $\mathrm{Pu}$, and Logan Carpenter, they investigated the problems of service scheduling for drones and Zone Service 
Providers (ZSPs) in the Internet of Drones. For performance evaluation, they conducted extensive simulation experiments using OMNeT++.

In [15] Guan, et al. they studies distributed algorithms for controlling self-organizing flying Unmanned Aerial Vehicles with massive MIMO networking capabilities, dubbed mDroneNet. The distributed solution algorithm converges in tens of iterations and can achieve around $90 \%$ of the global optimum, according to the Results.

In [16] Verri, et al. they discussed the drones' overall performance. They proposed a different approach to the problem of airspace route planning. They used a simplified simulation model.

In [17] Haas, and Zhong, they discussed the collaborative communication of multiple drones, as to create a reliable and powerful communication links, which as part of the network (they called "Network in the Sky").

In [18] Valianti, et al. they proposed Using a swarm of pursuer drones to track down and apprehend one or more rogue drones. Extensive performance assessment results show that the problem can be solved efficiently and effectively using the evolved distributed algorithm.

In [19] Khosravi, et al. they proposed designing drones trajectories that efficiently perform some transportation operation (for example, package delivery) while also providing uniform coverage over a neighbourhood area, which is required for applications such as network coverage, data collection from Internet of Things devices, wireless power transfer, and surveillance. They proposed a trajectory process that would allow for uniform coverage while maintaining transport (delivery) efficiency.

In [20] Dao, et al. they studied the Aerial radio access networks (ARANs) as a potential strategy to supplement existing terrestrial communication systems. Unmanned aerial vehicles, drones, and satellites are among the airborne components involved. The development of seamless mobile communication systems is expected to be aided by ARANs as part of a comprehensive sixth-generation (6G) global access infrastructure.

In [21] Real, et al. they presented a group of multiple Drones that would work together on autonomous construction missions. They tested and simulated the system's performance.

In [22] Huang, et al. they illustrated how to navigate a group of unmanned aerial vehicles (UAVs) to monitor traffic on a road. They tested their method using computer simulations.

\section{A. $5 \mathrm{~g}$ Mobile Communication System}

In [23] Habibi, et al. they discussed the fifth generation of mobile communication systems aims to provide a ubiquitous mobile service with better quality (QoS). Automobiles, public transportation, medical care, energy, public safety, agriculture, entertainment, manufacturing, and other vertical industrial applications are expected to benefit from this technology. User density, traffic volume, and data rate are all expected to increase dramatically. In the coming decade, innovative solutions to the needs of both smartphone users and vertical industries are needed, as shown in Fig. 1.

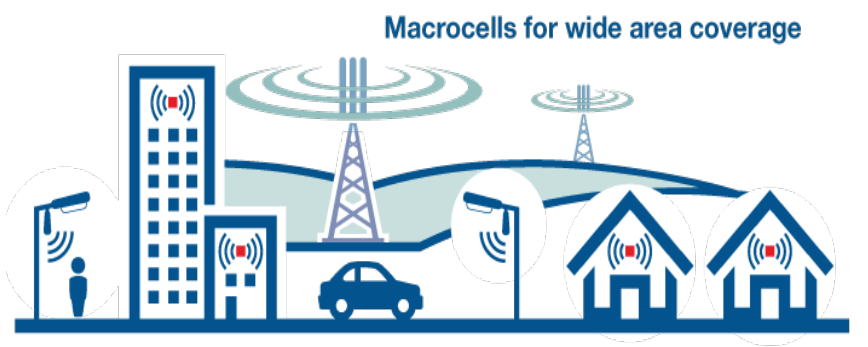

In-building and street small cells

Home small cells

Fig. 1. Abundance of the Antennas that the 5th Generation Networks Relay on.

\section{B. Ordinary Site Survey}

The human power is the main factor in the traditional way of measuring the signal strength of the 5th generation mobile networks. Lots of specialists work using their smartphones (using mobile application like "Network Cell info Lite") [23]. They are all responsible for making that process get done. Like going to all the places and coverage areas, measuring the signal strength and even directing the antennas on the base stations by themselves. They communicate with each other to send and receive the measures and the required data to control and direct the antennas.

So according to all that lots of problems occurred through this process like the high cost, high consumption of time and the lack of accuracy. Plus the process needs to get repeated so many times in small period of time to reach the direction required. Which is not achieved at the end.

\section{Image Processing}

In [24] Chen, et al. they framed this drone-view building identification as building retrieval problem: given a building (multimodal query) with its images, geolocation and drone's current location, they aim to retrieve the most likely proposal (building candidate) on a drone-view image. Despite few annotated drone-view images to date, there are many images of other views from the Web, like ground-level, street-view and aerial images. Thus, they proposed a cross-view triplet neural network to learn visual similarity between drone-view and other views.

\section{Mobile Application (To Measure Signal Strength)}

Will be measure the signal Strength by "Network Cell Info lite app" [23]. It will measure the signal strength and display the location of your signal on a map.

\section{E. MQTT Protocol/MQTT Cloud}

In [25] Kang, et al. they discussed MQTT protocol operation. The basic concepts of it is publish/subscribe and client/broker and its basic functionality is connect, publish, and subscribe.

In [26] Mishra and Attila, they discussed MQTT protocol, For message delivery, MQTT provides three levels for quality of service (QoS 0 - QoS 1 - QoS 2). The design principles of this protocol focus on minimizing network bandwidth and device resource requirements ensuring reliable delivery. 
In [27] presented by Krishna, et al. they discussed MQTT Dashboard, a mobile app that runs on a phone and can be used to track data sent from sensor nodes using the MQTT protocol. MQTT Dash is another choice. M2M, Arduino, Raspberry Pi, Microcontrollers, and other devices are supported by the application.

\section{F. Hardware Implementation}

In [28] Mathur, et al. proposed a novel approach to digital image processing by using the histogram of oriented gradients (HOG) features descriptor with the OpenCV library, which was programmed in Python and booted with the Raspberry $\mathrm{Pi}$, which was equipped with a RaspyiCam to capture moving images of objects passing under the cam.

\section{Overall Proposed Model}

As shown in Fig. 2. "Proposed Model" the human power has been replaced with "Drones" by creating a cooperative network "that based on new communication protocol between drones. A Cooperative Network between Drones will be built and a "4G Mobile" will be setuped and a "Raspberry pi" will be setuped on every drone. digital map will be used with drones so drones are ready to be sent to the locations to take the measures of the signal strength . All buildings will be identified by the " (BIS) ". The signal strength will be measured by a mobile application "Network Cell Info Lite" setuped on the "4G mobile". Data will be sent from "Drones" to "Server" and vice versa using Cooperative network based on a new communication protocol and also the messages will be sent between "Drones" and" Server" and vice versa by "MQTT Cloud". So, Finally the "Server" will control the direction of the antennas using "Servo motors".

The Model Structure:

1) Building cooperative network between drones: "Drones" will communicate with each others and Data will be sent from "Drones" to "Server" and vice versa using Cooperative network based on our new designed communication protocol, as shown in Section 5.

2) Building new communication protocol: The Drones communicate with the Server or other Drones is realized in defined frame structure of new communication protocol supporting cooperative network, which consists of (payload data, header) for identification. The frame structure consists of the (header, payload) accompanied by the checksum. The protocol is a designed communication protocol to support the cooperative networks between drones to be used in 5G site survey.
3) Mobile app SW (network cell info lite): It will measure the signal strength and display the location of your signal on a map.

4) Building Identification System (BIS) on drones: As shown in [24]. The work of the "BIS based on CNN" is to give every building a specific number to recognize the entrance of the building to measure signal strength at every floor.

5) Directing antenna system (DAS: The (DAS) will be setuped on "Raspberry pi" and programmed using "Python language". It will receive messages "with measures "from "Drones" and according to these measures, the parameters of the angles of the antennas will be given to the three groups of "Servo motors" so that the antennas will be directed to the optimal direction.

6) MQTT (broker/clint - publisher/subscriber): "Drones" will communicate with each others and with the antennas through exchanging messages by "MQTT cloud".

7) Drones: The "4G Mobile" and "Raspberry pi" will be setuped on Drones, then the signal strength measured will be sent to the base station by the drones.

8) Phones: Setup the "4G Mobile" on Drones and Setup a mobile App (Network Cell Info Lite - Mobile \& Wi-Fi Signal) on smart phone "4G Mobile" to measure signal Strength.

9) Raspberry Pi: This versatile single board machine can be used for a variety of tasks. two "Raspberry pis" will be used : First with an (DAS) and second with an (BIS).

10)Servo motors: The designed system requires maximum precision, in 5G, servo motors will be used to control antenna direction. The Server will give orders to the three groups of "Servo Motors" to control the direction of the antennas (every group consists of 2 servo motors to move one antenna "Pan1, Tilt1").

As shown in Fig. 3. "Proposed model flow chart" A Cooperative Network will be built between Drones and a "4G Mobile" and a "Raspberry pi" will be setuped on all drones. The digital map will be used on Drones so they are ready to be sent to their locations for taking the measures of the signal strength. The "(BIS)" will identify all buildings. Then the signal strength will be measured by a mobile application like "Network Cell Info Lite" which setuped on the "4G mobiles". Data will be sent from "Drones" to "Server" and vice versa by Cooperative network based on a new communication protocol and also the messages will be sent between "Drones" and" Server" and vice versa by "MQTT Cloud". So at the end the "Server" will control the direction of all antennas using "Servo motors". And the process will be repeated again and again till achieve our target. 


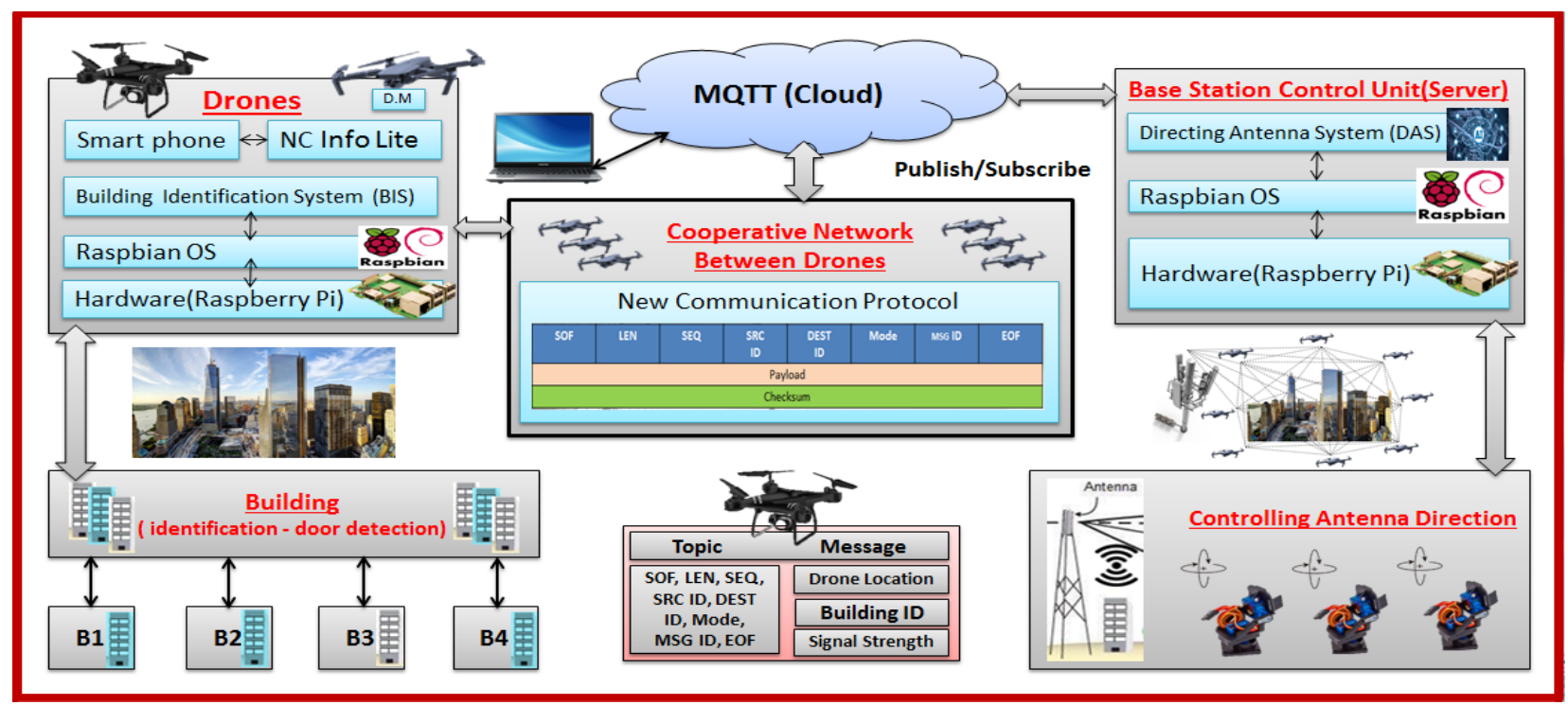

Fig. 2. Proposed Model.

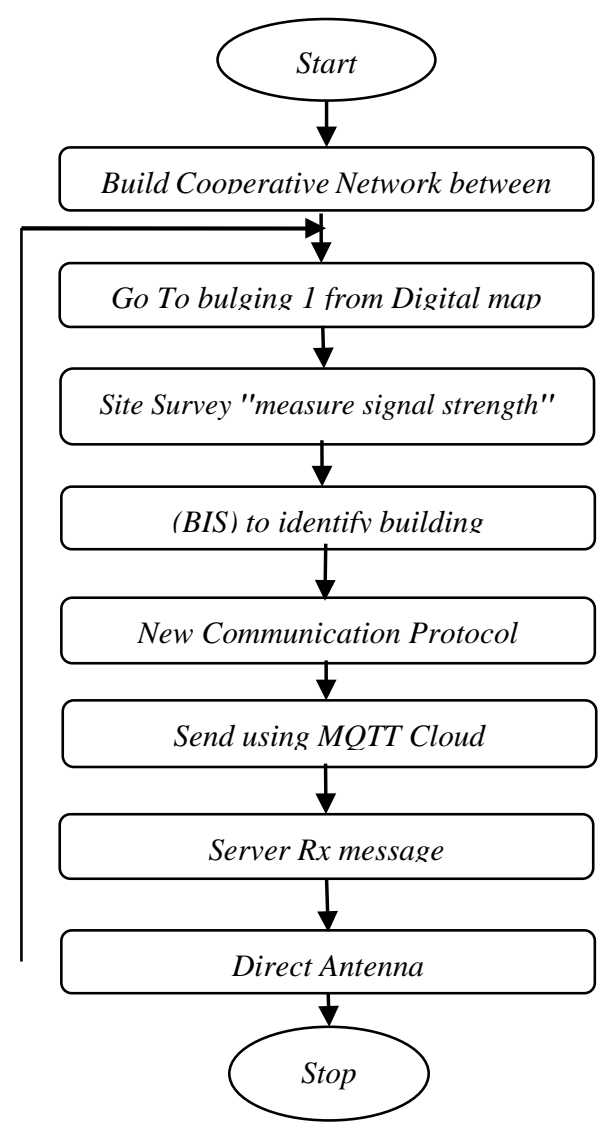

Fig. 3. Proposed Model Flow Chart.

\section{Drones CoOperative Network}

As shown in Fig. 4, the Cooperative Network: (Peer to Peer) in a P2P network, the "peers" are Drone which are connected to each other via the Cooperative network. Drones can be connected directly with other drones in the network without the need of a Master through MQTT Cloud through an internet connection.

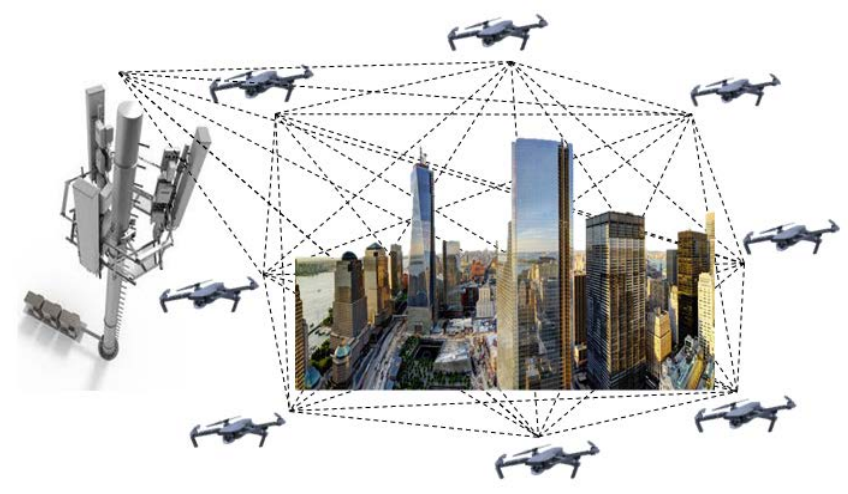

Fig. 4. Peer-to-Peer Cooperative Network between Drones.

\section{Proposed New Communication Protocol}

- In this paper, a comprehensive solution was proposed by design a new communication protocol to support the cooperative networks between drones to be used in different fields and applications."Drones" will communicate with each others and with the antennas through exchanging messages by "MQTT cloud".

- MQTT has three components (Publisher "Drones/Server", Subscriber "Drones/Server", Broker MQTT Cloud).

- Mosquitto MQTT is used as a broker.

- The Drones communicate with the Server or other Drones is realized in defined frame structure of new communication protocol supporting cooperative network, which consists of (payload data, header) for identification. The frame structure consists of the (header, payload) accompanied by the checksum.

- The frame structure of the new communication protocol is shown in Fig. 5. 


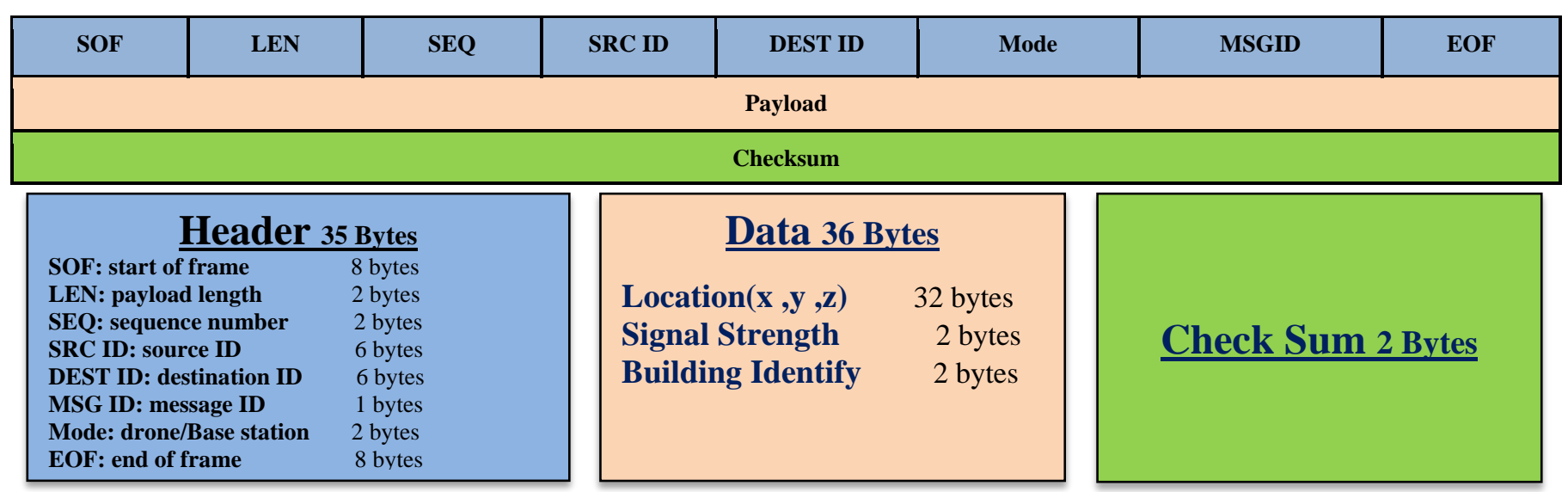

Fig. 5. Communication Frame Structure.

The frame structure fields will be explained in the following below:

a) Header: The protocol is including a header that contains all of the information about the payload data that must be exchanged between the Drones and the Server (Base Station antenna's) or Drones and Drones. The header is of 35 bytes, it has a set length and consists of the fields mentioned below.

- SOF: In continuous transmission, this Start of Frame implies the beginning of a frame. The field length is 8 Bytes. which indicates the beginning of new packet.

- EOF: In continuous transmission, this End of Frame indicates the end of a frame. The field is 8 bytes long. This indicates that the previous frame has come to an end.

- LEN: The length of the payload is indicated by this value. (Location( $\mathrm{x}, \mathrm{y}, \mathrm{z}$ )/Signal Strength/Building Identify) will be the payload, payload length max 7200 bytes which uses 2 bytes, (signal strength is 2 bytes and Building Identity is 2 bytes and GPS data is 32 bytes, taking 200 readings for each building with total of 36 * $200=7200$ bytes), The original data or message to be sent is referred to as the payload.

- SEQ: The 2 byte Sequence The long message is divided into a number of packets and numbered, allowing it to be assembled at the receiving end.

- SRC ID: Source identification is used to determine the system's uniqueness among the various Drones that are connected to one another. source ID has a length of 6 bytes.

- DEST ID: The receiving destination's address is stored in the Destination ID field, destination ID represents Drone/Server number. The length of the destination ID is 6 bytes. By including the data's destination address, it can be ensured that the data intended for a specific drone arrives there and others drone may reject them.

- MSG ID: 1 byte Message Identification of the parameter is used to distinguish parameters from the many that are available (control msg/cooperative
network/Drones and the Server). Each of these parameters has a unique ID prefix, which aids in faster and more reliable communication.

- MODE: send to drone or base station 2 bytes, indicating the number of Drones in swarm flight .

b) Payload: This is the original flight data, Payload of one data information is : 36 bytes.

(Navigation information of drone and camera information is not included in new communication protocol).

c) Checksum 2byte: This is used to verify the data's integrity. At the transmitter, the frame's checksum is calculated and appended to the frame at a specific location in the frame structure. The checksum for the received frame will be calculated and compared to the value that comes with the frame. A value mismatch between the Transmitter and the Receiver indicates that the data is incorrect and frame should be discarded, with a request for re-transmission. ITU X.25 is used to calculate the frame's checksum [8].

- There is an example of a one "Drone" sending a message from a drone to the server, message consists of three frames. Each frame measures the signal strength from a different position. (Server ID: 4, Drone 1 ID: 1 , No. of frames: 3 ) as shown in Table I.

Header is 35 bytes

(SOF: 8 bytes, EOF: 8 bytes, LEN: 2 bytes, SEQ: 2 bytes, SRC ID: 6 bytes, DEST ID: 6 bytes, MSG ID: 1 byte, Mode: 2 bytes)

Payload of one data information is 36 bytes

(Building Identify 2 bytes, Signal Strength 2 bytes, GPS location of Drone (x, y, z) is 32 bytes)

Checksum is 2 bytes

The size of Message $=$ total size of frames $=($ frame size $*$ number of frames)

The size of one frame $=$ Header size + Payload size + Checksum size $=35+36+2=73$ Bytes

The size of Message $=73 * 3=219$ Bytes. 
TABLE I. EXAMPLE FOR TRANSLATE THE DATA

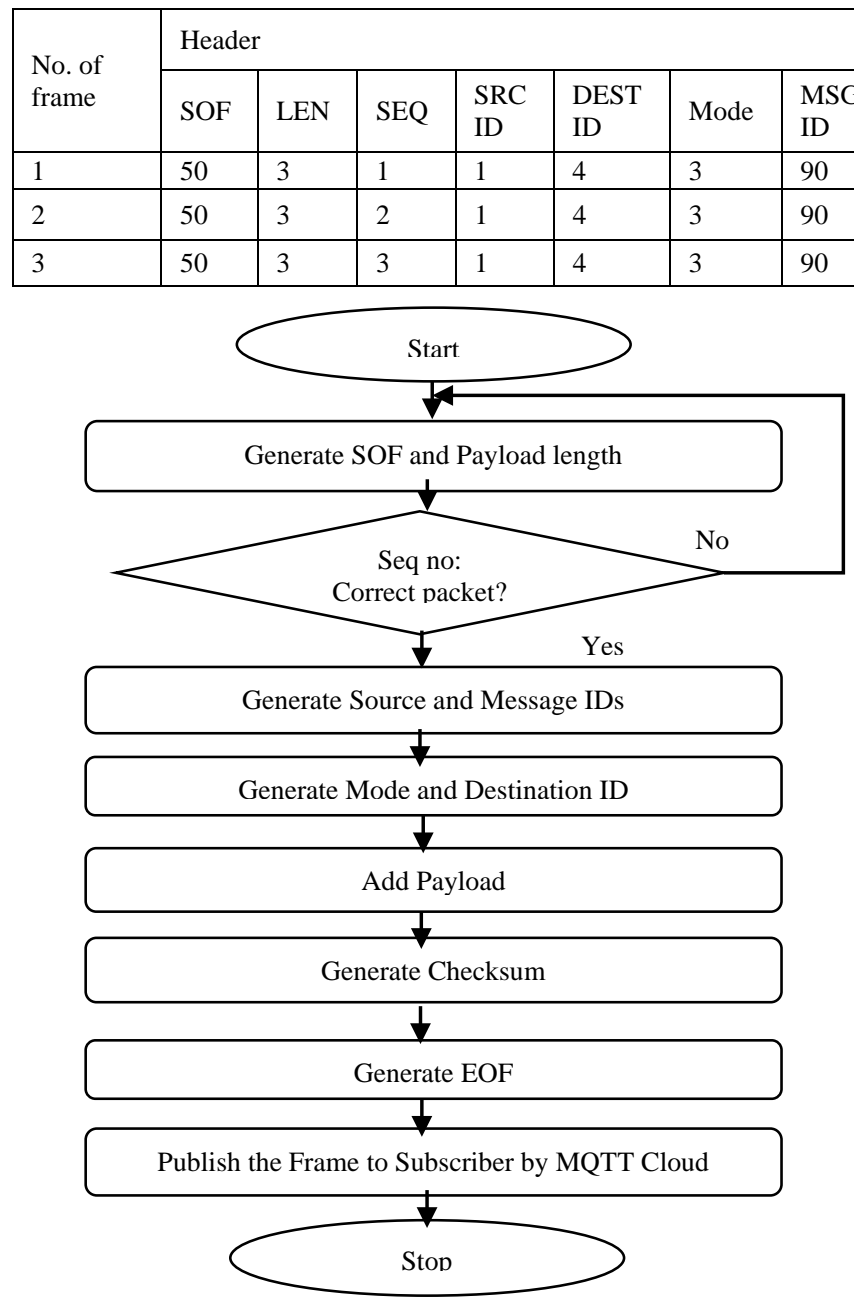

Fig. 6. Flowchart of the Publisher.

As shown in Fig. 6. "Flowchart of the Publisher" at the transmitter "publisher" side, a fixed SOF indicates the start of new packet. Once the payload data is arrived for first time, the SEQ field is initializes and length of the payload is calculated and fed into the LEN field in the frame. The SRC ID and DEST ID is generated and appended to the frame. MODE is decided and appropriate value is appended in the frame, to this header, the payload data is added and checksum is calculated for the entire frame and a fixed EOF indicates the End of packet and appended at the last position of the frame. Now the frame is ready for transmission "publish" to the MQTT Cloud.

As shown in Fig. 7. "Flowchart of the Subscriber" At the receiver "Subscriber" side the decoding procedure is followed as given in the Fig. 9. Subscribe the Frame at the Receiver from "MQTT Cloud" Once the packet is received, it is cross checked with the transmitted checksum to check the integrity of the transmitted data. If the checksum value matches the transmitted ones, the corresponding payload parameter is parsed by extracting the corresponding IDs. The DEST ID is checked with the host ID to check the transmitted packet is intended for host or not. A mismatch in checksum indicates the packet wrong data.

\begin{tabular}{|c|c|c|c|c|c|c|}
\hline \multirow{3}{*}{$\begin{array}{l}\text { EOF } \\
500\end{array}$} & \multicolumn{5}{|c|}{ Payload } & \multirow{3}{*}{$\begin{array}{l}\text { Check } \\
\text { Sum } \\
2304\end{array}$} \\
\hline & \multicolumn{3}{|c|}{ Location( $\mathrm{x}, \mathrm{y}, \mathrm{z}$ ) } & $\begin{array}{l}\text { Signal } \\
\text { Strenoth }\end{array}$ & Building & \\
\hline & 398 & 195 & 104 & 1757 & B1 & \\
\hline 500 & 52 & 451 & 91 & 3843 & B1 & 2349 \\
\hline 500 & 423 & 97 & 250 & 7331 & B1 & 2973 \\
\hline
\end{tabular}

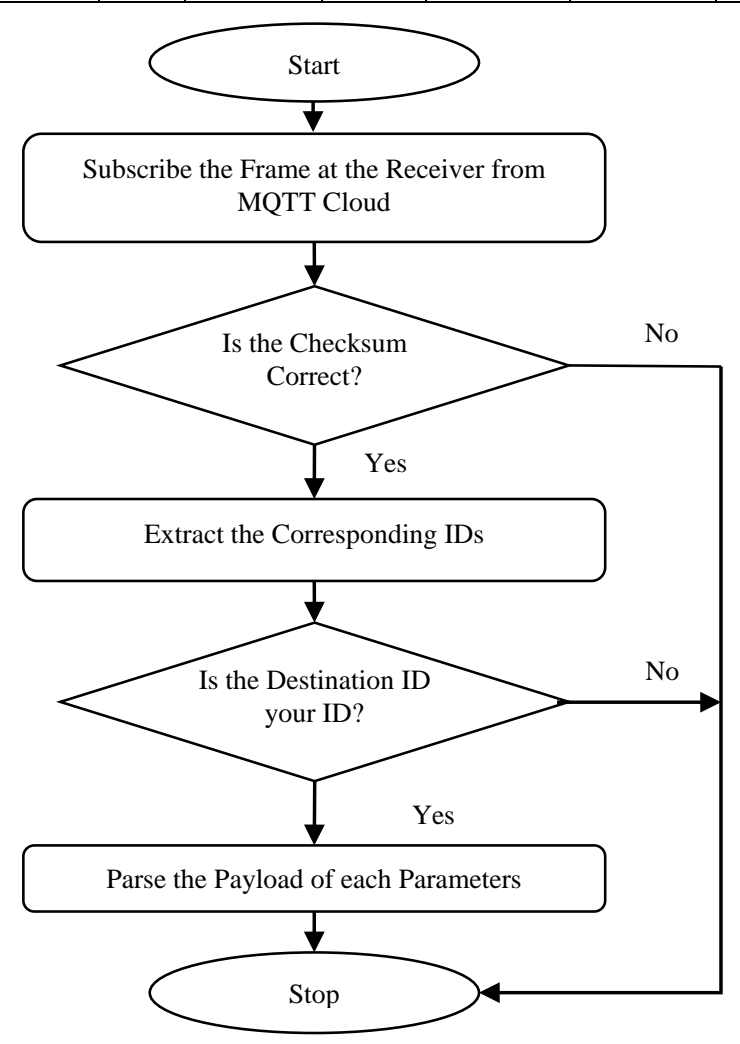

Fig. 7. Flowchart of the Subscriber.

\section{Mobile ApplicAtion}

Network Cell Info Lite - Mobile \& WiFi Signal (To measure signal Strength), with testing cell signal strength, it's easier to see how subtle differences in signal strength can affect the performance of the mobile communication system. Lots of people actually consider "Network Cell Info lite app" the best of all apps to measure signal Strength. And for good reason. It covers every cellular network.

\section{BUILDING IDENTIFICATION SYSTEM (BIS) AND (DAS)}

\section{A. Proposed Building Identification System}

Fig. 8 shows the steps of Building Identification System of detecting the building entrance as shown in [24], which setuped on drone for building identification. The Raspberry Pi model "zero w" will be run by Rasbian OS, Install Building Identification System based on CNN using Python and Install OpenCV for Building Identification. First, send digital map to drones to be able to match the building image with it, then generate "SOF, LEN, SEQ", then check the SEQ no, then generate "SRC ID, MSG ID, Mode, DEST ID, Checksum, and EOF". then creating Cooperative Network between Drones 
then detect the drone location then proceed to make the Building Identification by giving the OpenCV sample of Buildings photo as a training sample and matching the building image with digital map, then read the measures of the signal strength by "NC Info Lite app" which setuped on Smart phone, and finally will Publish the frames on "MQTT Cloud".

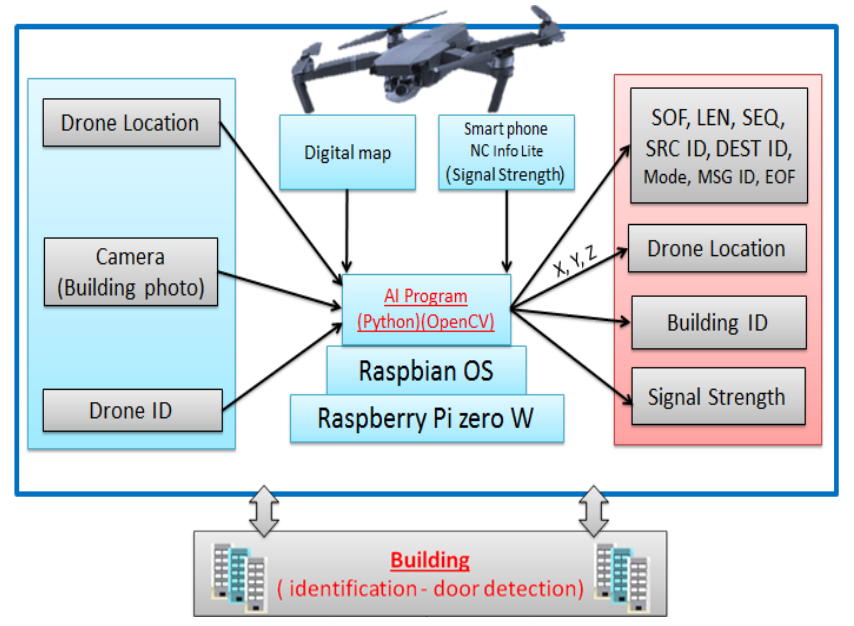

Fig. 8. Implemented Building Identification System.

\begin{tabular}{cc}
\hline Algorithm 1: The algorithm for Building Identification System to \\
detect building entrance
\end{tabular}

\section{B. Proposed Directing Antenna System (DAS)}

Fig. 9 shows Directing Antenna System. Which setuped on "Raspberry Pi" on the base station to control the antennas, the Raspberry Pi will be run by Rasbian OS, Install (DAS) using Python to control antenna.

First, (DAS) will subscribe the Frame information, payload, and checksum value from "MQTT Cloud" that has sent from (BIS) setuped on Drones, then generate checksum value and match the checksum value received from drones with the checksum value generated. If they matched, the corresponding IDs will be extracted. And if not, the process will be repeated again (subscribe the Frame information, payload, and checksum value from MQTT Cloud). Then generate servo motors degrees (Pan1, Tilt1, Pan2, Tilt2, Pan3, Tilt3) based on the measures of the signal strength to be sent to servo motors.

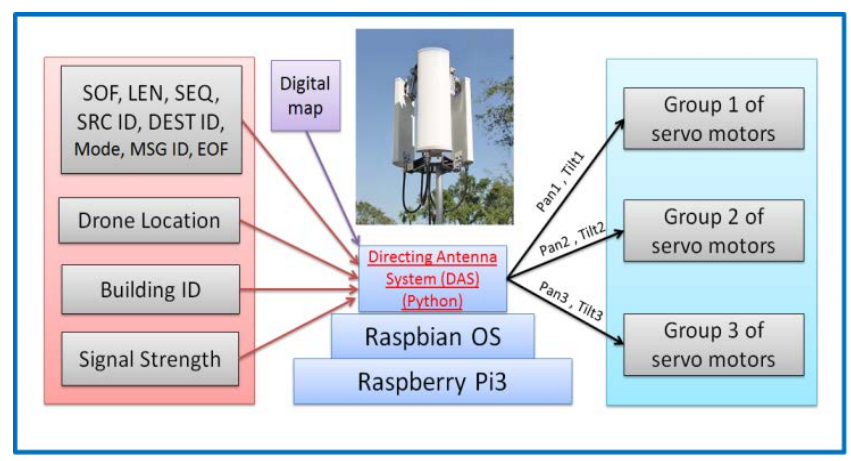

Fig. 9. (DAS) to Control Antenna.

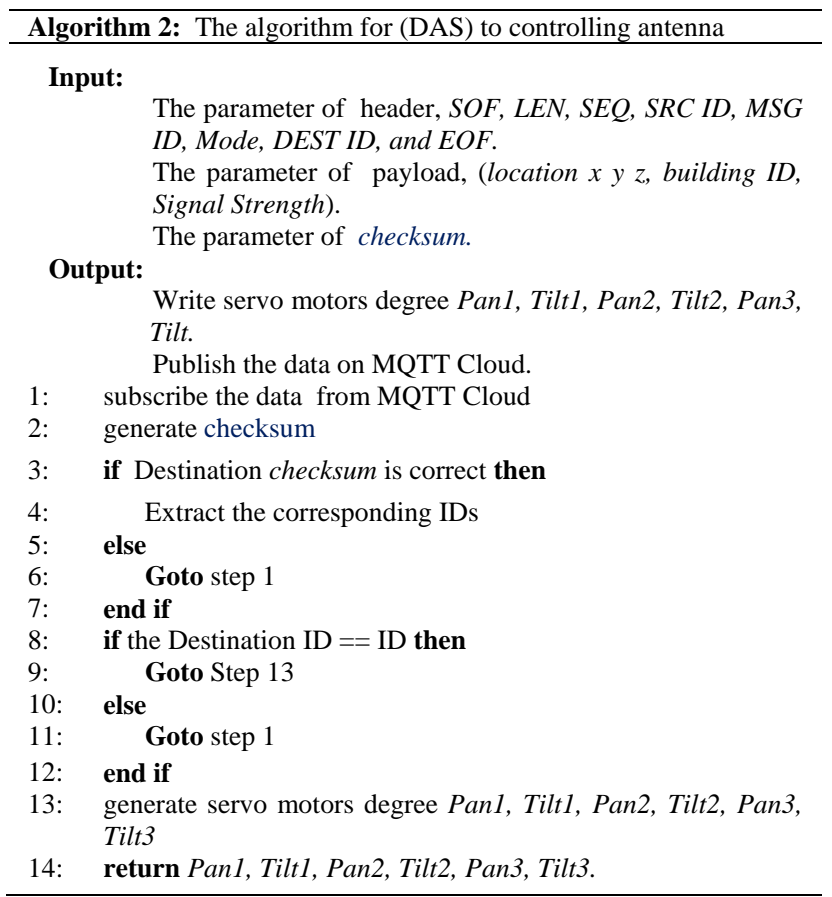

VIII. Simulation Results AND PERFormanCE ANAlysis

- Simulation tools

The simulation experiment is to verify the feasibility and performance of the communication protocol and the system. In order to validate our work, OMNeT++ (Objective Modular Network Testbed in C++) framework will be used. OMNET++ is an open source discrete event simulator with modular, component-based C++ simulation library and framework. [29]

The implementation leverages a series of shared libraries over INET, an OMNeT++ model suite for the simulation of wired, wireless and mobile networking protocols. [30].

- Simulation scenario and results

Three Drones are used and there is a cooperation network between them. And through the 2 antennas, the signals will be sent between Drones and the server. Drones measure the signal strength within all the buildings areas and send it to the server 
to direct the antennas to the optimal direction. Then measuring the network throughput.

The video in this link shows our simulation scenario

https://drive.google.com/file/d/1KLneJvXlqTj2ZZqFvQWzEzY_o u06y4Bh/view?usp=sharing

Bandwidth is measured for nine different bandwidth throttles within the speed range of [1 - 16] Mbps downlink and [1- 5] Mbps uplink, and data size 0.11138916 Mbits.

The Data Size is calculated as the following equation where The Data Size (in Mbits) equals the size of the frame (in byte) multiplied by 8 bits to convert it to bits, then multiply size of the frame (in bits) by the number of reading, And then divide by 1024/1024 to convert to Mbits, after that the result will be multiplied by the number of Buildings.

Data Size (Mbits) $=((($ Size of frame $($ Byte $) * 8$ bits * reading) / $\mathrm{KB} / \mathrm{MB}) *$ for one Building)

Mbits

$(((73 * 8 * 200) / 1024$ / 1024) * 1) $=0.11138916$

Time_Downlink Throughputs = Data size (Megabits) $/$ Downlink $($ Mbps $)=(\mathrm{s})$

Time_uplink Throughputs $=$ Data size $($ Megabits) $/$ uplink $(\mathrm{Mbps})=(\mathrm{s})$

Network throughput is one of the key network performance metrics for QoS among end-users. However, demonstrating reliability and validity of network throughput measurement deployed in vendor end-point is a critical task [31].

As shown in Fig. 10, the results of uplink and Downlink speed measurements. Each stripped Brick color bar represents the measured uplink speed that is starting to rise from 1 to 5 Mbps. Similarly, each blue bar represents downlink throughput measurements. The speed is constantly increasing as the Bandwidth increases. that is starting to rise from 1 to $16 \mathrm{Mbps}$.

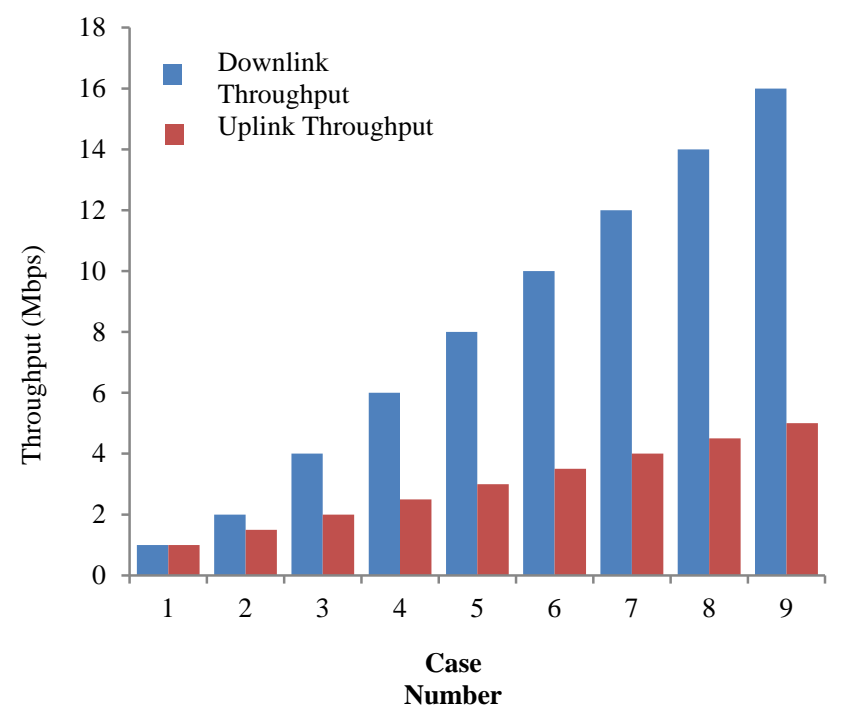

Fig. 10. Measured Downlink /uplink Throughputs for 9 Cases.

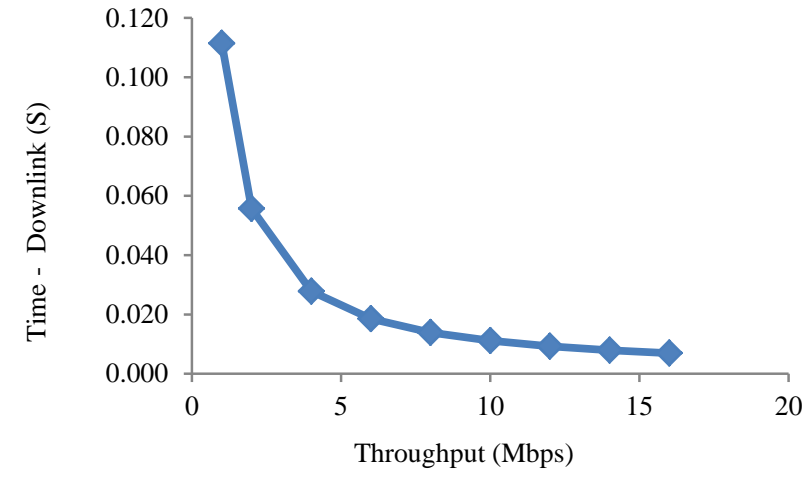

Fig. 11. Time_Downlink Throughputs.

Throughputs_Downlink

As shown in Fig. 11 the Downlink Time within the time range of [0.000 - 0.120] (S) for receiving the amount of data "0.11138916 Mbits" of measuring the signal strength for one building in nine different bandwidth throttles within the speed range of [1 - 16] Mbps. In order to better demonstrate accuracy of the results, will depicting overlay line diagrams for Downlink Time of 9 Cases results.

The figure also illustrates the results of Downlink time and different values for Downlink speed measurements that start to rise from 1 to $16 \mathrm{Mbps}$. Each blue dot represents the measured downlink speed for the nine cases. The speed is constantly increasing as the Bandwidth increases, On the contrary, the Downlink time decreases as the Bandwidth increases. As shown in the previous example when the data size equal $0.11138916 \mathrm{Mb}$ and speed is $1 \mathrm{Mbps}$, time becomes $0.111389160 \mathrm{~S}$ while the speed increased to $16 \mathrm{Mbps}$, time decreased to $0.006961823 \mathrm{~S}$.

Throughputs_Uplink

As shown in Fig. 12, the Uplink Time within the time range of $[0.000$ - 0.120] (S) for sending the amount of data "0.11138916 Mbits" of measuring the signal strength for one building in nine different bandwidth throttles within the speed range of [1.0 - 5.0] Mbps. In order to better demonstrate accuracy of the results, will depicting overlay line diagrams for Uplink Time of 9 Cases results.

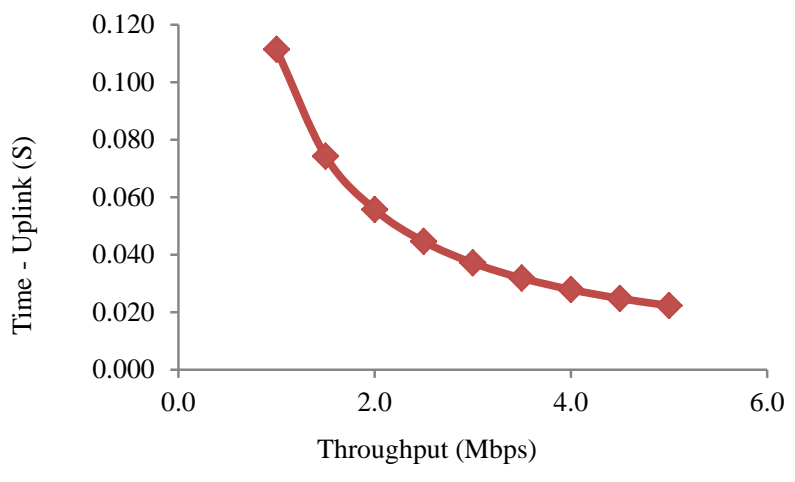

Fig. 12. Time_uplink Throughputs. 
The figure also illustrates the results of Uplink time and different values for Uplink speed measurements that start to rise from 1 to 16 Mbps. Each red dot represents the measured uplink speed for the nine cases. The speed is constantly increases as the Bandwidth increases, On the contrary, the Downlink time decreases as the Bandwidth increases. As shown in the previous example when the data size equal $0.11138916 \mathrm{Mb}$ and the speed is $1 \mathrm{Mbps}$, time becomes $0.111389160 \mathrm{~S}$ but when the speed increased to $5 \mathrm{Mbps}$, time decreased to $0.022277832 \mathrm{~S}$.

As shown in these figures, the results are significantly correlated with each other and can support each other which advocate validity of the performance evaluations.

\section{HARDWARE IMPLEMENTATION}

In order to position the antennas, "I ${ }^{2} \mathrm{C}$ Servo Driver" will be used (using $\mathrm{I}^{2} \mathrm{C}$ protocol) connected to "Raspberry pi" (which used as a server) to control all groups of servo motors which are responsible for fully control and direct the antennas. There is three groups of "Servo motors". Every group consists of two "Servo motors" to control the antennas with PAN/TILT mechanism. "Raspberry bi" will give orders to the groups of "Servo Motors" to direct the antennas till it reachs the optimal direction.
Raspberry Pi as a Server. The I2C will be used as a communication protocol between the Server and the servo motors, and Three Groups of servo motors to controlling in three antennas, each group consist of two servo motor (Pan , Tilt).

- The prototype to control antenna

There are three groups of "Servo motors". Every group consists of 2 "Servo motors" to control the antennas with PAN/TILT mechanism. "Raspberry bi" will give orders to the groups of "Servo Motors" to direct the antennas till it reaches the optimal direction.

The video in this link shows our prototype for part of Hardware Implementation

https://drive.google.com/file/d/1xqOLXLoxJJDSuD7dtEJy FQNdcIrfaSI2/view?usp=sharing

\section{COMPARISON WITH OTHER WORKS}

"To the best of our knowledge, our proposal is the first work to control the antennas direction of mobile communication using drones", the Table II shows the limitations of the previous communication protocol and cooperative network between drones against the new proposed work.

TABLE II. The Limitations of the Previous Works Against the NeW Proposed Work

\begin{tabular}{|c|c|c|c|c|c|c|c|c|}
\hline $\begin{array}{l}\text { Reference } \\
\text { No. }\end{array}$ & subject (problem) & $\begin{array}{l}\text { Communication } \\
\text { Protocol }\end{array}$ & $\begin{array}{l}\text { Throughput } \\
\text { measuring }\end{array}$ & $\begin{array}{l}\text { Packet } \\
\text { Reception } \\
\text { Ratio }\end{array}$ & $\begin{array}{l}\text { Cooperative } \\
\text { Network }\end{array}$ & Simulation & $\begin{array}{l}\text { SW } \\
\text { system }\end{array}$ & $\begin{array}{l}\text { HW } \\
\text { Implementation }\end{array}$ \\
\hline $\begin{array}{l}\text { The } \\
\text { proposed } \\
\text { work }\end{array}$ & 5G site survey & $\sqrt{ }$ & $\sqrt{ }$ & $\mathrm{x}$ & $\sqrt{ }$ & $\left(\right.$ Omnet $\left.^{++}\right)$ & DAS / BIS & $\sqrt{ }$ \\
\hline [1] & Mobile Communications & $\mathrm{x}$ & $\mathrm{x}$ & $\sqrt{ }$ & $\mathrm{x}$ & $\mathrm{x}$ & $\mathrm{x}$ & $\mathrm{x}$ \\
\hline [3] & Intrusions detection & $\mathrm{x}$ & $\mathrm{x}$ & $\mathrm{x}$ & $($ Swarm $) \sqrt{ }$ & $\left(\right.$ Omnet $\left.^{++}\right)$ & $\mathrm{x}$ & $\mathrm{x}$ \\
\hline [4] & $\begin{array}{l}\text { disaster, rescue, } \\
\text { aerial monitoring }\end{array}$ & $\mathrm{x}$ & $\mathrm{x}$ & $\mathrm{x}$ & $\sqrt{ }$ & $\mathrm{x}$ & $\sqrt{ }$ & $\sqrt{ }$ \\
\hline [5] & $\begin{array}{l}\text { Moving Targets } \\
\text { Surveillance }\end{array}$ & $\mathrm{x}$ & $\mathrm{x}$ & $\mathrm{x}$ & $\sqrt{ }$ & $\sqrt{ }$ & $\mathrm{x}$ & $\sqrt{ }$ \\
\hline [6] & $\begin{array}{l}\text { Coverage of Drone Small } \\
\text { Cells }\end{array}$ & $\mathrm{x}$ & $\mathrm{x}$ & $\mathrm{x}$ & $\sqrt{ }$ & $($ Matlab) $\sqrt{ }$ & $\mathrm{x}$ & $\sqrt{ }$ \\
\hline [8] & $\begin{array}{l}\text { communication between } \\
\text { Ground Station and drones }\end{array}$ & $\sqrt{ }$ & $\mathrm{x}$ & $\mathrm{x}$ & $\begin{array}{l}\text { (master/slaves) } \\
\mathrm{x}\end{array}$ & $\mathrm{x}$ & $\mathrm{x}$ & $\sqrt{ }$ \\
\hline [9] & Parcel delivery & $\mathrm{x}$ & $\mathrm{x}$ & $\mathrm{x}$ & $\begin{array}{l}\text { (Multi- } \\
\text { Drones) } \mathrm{x}\end{array}$ & $\sqrt{ }$ & $\mathrm{x}$ & $\mathrm{x}$ \\
\hline [10] & $\begin{array}{l}\text { Interference in Pipeline } \\
\text { Networks }\end{array}$ & $\mathrm{x}$ & $\mathrm{x}$ & $\mathrm{x}$ & $\sqrt{ }$ & $($ Matlab) $\sqrt{ }$ & $\mathrm{x}$ & $\mathrm{x}$ \\
\hline [11] & radio base station inspection & $\mathrm{x}$ & $\mathrm{x}$ & $\mathrm{x}$ & $\sqrt{ }$ & $(\mathrm{UNITY}) \sqrt{ }$ & $\sqrt{ }$ & $\sqrt{ }$ \\
\hline [12] & $\begin{array}{l}\text { positioning measuring in } \\
\text { IOD }\end{array}$ & $\mathrm{x}$ & $\mathrm{x}$ & $\mathrm{x}$ & $\sqrt{ }$ & $\sqrt{ }$ & $\mathrm{x}$ & $\mathrm{x}$ \\
\hline [13] & Assigning location & $\mathrm{x}$ & $\mathrm{x}$ & $\mathrm{x}$ & $\begin{array}{l}\text { (Multi- } \\
\text { Drones) } \mathrm{x}\end{array}$ & $\sqrt{ }$ & $\mathrm{x}$ & $\mathrm{x}$ \\
\hline [18] & Disabling a Rogue Drone & $\mathrm{x}$ & $\mathrm{x}$ & $\mathrm{x}$ & $\sqrt{ }$ & $\sqrt{ }$ & $\mathrm{x}$ & $\mathrm{x}$ \\
\hline [19] & Transport Applications & $\mathrm{x}$ & $\mathrm{x}$ & $\mathrm{x}$ & $\begin{array}{l}\text { (Multi- } \\
\text { Drones) } \mathrm{x}\end{array}$ & $\sqrt{ }$ & $\mathrm{x}$ & $\mathrm{x}$ \\
\hline [21] & Cooperative Construction & $\mathrm{x}$ & $\mathrm{x}$ & $\mathrm{x}$ & $\sqrt{ }$ & $\sqrt{ }$ & $\sqrt{ }$ & $\sqrt{ }$ \\
\hline [22] & monitor the traffic & $\mathrm{x}$ & $\mathrm{x}$ & $\mathrm{x}$ & (Network) $\sqrt{ }$ & $\sqrt{ }$ & $\mathrm{x}$ & $\mathrm{x}$ \\
\hline
\end{tabular}


TABLE III. PROPOSED MOdEL Against Traditional Work

\begin{tabular}{|l|l|l|l|}
\hline \multicolumn{2}{|l|}{} & The proposed work & Traditional way \\
\hline 1 & Time & Hours & Weeks \\
\hline 2 & Accuracy & Accurate & Not accurate \\
\hline 3 & Human Power & No persons & Lot of persons \\
\hline 4 & Cost & Low cost & High cost \\
\hline
\end{tabular}

As a result all the disadvantages that mentioned before, As shown in Table III, will be avoided and we will no longer face any high in the cost caused by repeating the process in the traditional way.

\section{CONCLUSION AND FUtURE WORK}

In this paper, a comprehensive solution was proposed by designing a standard communication protocol to support the cooperative networks between drones to be used in different fields \& applications. The system is for controlling the antenna direction of mobile communication using drones to resolve the problem as the $5 \mathrm{G}$ mobile users on higher floors of high buildings suffer from the weak signals. The proposed model included new communication protocol, cooperative network between drones, Building Identification System (BIS) to detect building entrance based on CNN, Directing Antenna System (DAS) to control antenna and hardware controlling antenna. the "Python Programing language" is used for Programing the (BIS and DAS), and " Raspberry Pi" platform to implement the system, and "Raspbian" operating system for " Raspberry Pi" and OpenCV, CNN algorithm for the Building Identification and give the OpenCV sample of Buildings photos as a training sample. MQTT Cloud used to transfer data between drones and server, "OMNeT++" for making the simulation, and the performance of the new Communication Protocol and Network throughput will be measured. At the end we will no longer face the disadvantages like the high cost, the high consumption of time and the lack of accuracy.

Build more cooperative Network to use Applications Between Drones, and Secure the New Proposed Communication Protocol of Drones Cooperative Network.

\section{REFERENCES}

[1] Ansari, Nirwan, and Liang Zhang. "Flexible backhaul-aware DBS-aided HetNet with IBFD communications." ICT Express 6.1 (2020): 48-56.

[2] Zeadally, S., J. Guerrero, and J. Contreras. "A tutorial survey on vehicleto-vehicle communications." Telecommunication Systems 73.3 (2020): 469-489.

[3] Bekhti, Mustapha, Nadjib Achir, and Khaled Boussetta. "Swarm of Networked Drones for Video Detection of Intrusions." International Wireless Internet Conference. Springer, Cham, 2017.

[4] Yanmaz, Evşen, et al. "Drone networks: Communications, coordination, and sensing." Ad Hoc Networks 68 (2018): 1-15.

[5] Gu, Jingjing, et al. "Multiple moving targets surveillance based on a cooperative network for multi-UAV." IEEE Communications Magazine 56.4 (2018): 82-89.

[6] Dong, Chao, et al. "Optimal deployment density for maximum coverage of drone small cells." China Communications 15.5 (2018): 25-40.
[7] Chakrabarty, Anjan, et al. "Vehicle to Vehicle (V2V) communication for Collision avoidance for Multi-copters flying in UTM-TCL4." AIAA Scitech 2019 Forum. 2019.

[8] Manasa, K. M., et al. "Design and development of communication protocol for micro air vehicle." 2015 Twelfth International Conference on Wireless and Optical Communications Networks (WOCN). IEEE, 2015.

[9] Iranmanesh, Saeid, et al. "Improving Throughput of 5G cellular Networks via 3D placement Optimization of Logistics Drones." IEEE Transactions on Vehicular Technology (2021).

[10] Ma, Dazhong, et al. "An Optimal Three-Dimensional Drone Layout Method for Maximum Signal Coverage and Minimum Interference in Complex Pipeline Networks." IEEE Transactions on Cybernetics (2021).

[11] Buksz, Dorian, et al. "Intent-driven strategic tactical planning for autonomous site inspection using cooperative drones." 2020 IEEE/RSJ International Conference on Intelligent Robots and Systems. IEEE, 2020.

[12] Lee, Chao-Yang. "Cooperative Drone Positioning Measuring in Internetof-Drones." 2020 IEEE 17th Annual Consumer Communications \& Networking Conference (CCNC). IEEE, 2020.

[13] Bartolini, Novella, Andrea Coletta, and Gaia Maselli. "A Multi-Trip Task Assignment for Early Target Inspection in Squads of Aerial Drones." IEEE Transactions on Mobile Computing (2020).

[14] Pu, Cong, and Logan Carpenter. "\$ Psched \$: A Priority-Based Service Scheduling Scheme for the Internet of Drones." IEEE Systems Journal (2020).

[15] Guan, Zhangyu, et al. "Distributed Joint Power, Association and Flight Control for Massive-MIMO Self-Organizing Flying Drones." IEEE/ACM Transactions on Networking 28.4 (2020): 14911505.

[16] Verri, Filipe Alves Neto, et al. "An Analysis on Tradable Permit Models for Last-Mile Delivery Drones." IEEE Access 8 (2020): 186279-186290.

[17] Haas, Zygmunt J., and Zhong Zheng. "Engineering a Network in the Sky." 2021 International Conference on Information Networking (ICOIN). IEEE, 2021.

[18] Valianti, Panayiota, et al. "Multi-Agent Coordinated Close-in Jamming for Disabling a Rogue Drone." IEEE Transactions on Mobile Computing (2021).

[19] Khosravi, Mohammadjavad, et al. "Multi-Purpose Drones for Coverage and Transport Applications." IEEE Transactions on Wireless Communications (2021).

[20] Dao, Nhu-Ngoc, et al. "Survey on Aerial Radio Access Networks: Toward a Comprehensive 6G Access Infrastructure." IEEE Communications Surveys \& Tutorials (2021).

[21] Real, Fran, et al. "Experimental Evaluation of a Team of Multiple Unmanned Aerial Vehicles for Cooperative Construction." IEEE Access 9: 6817-6835 (2021).

[22] Huang, Hailong, Andrey V. Savkin, and Chao Huang. "Decentralised Autonomous Navigation of a UAV Network for Road Traffic Monitoring." IEEE Transactions on Aerospace and Electronic Systems (2021).

[23] Habibi, Mohammad Asif, et al. "A comprehensive survey of RAN architectures toward 5G mobile communication system." IEEE Access 7 (2019): 70371-70421.

[24] Chen, Chun-Wei, et al. "Drone-View Building Identification by CrossView Visual Learning and Relative Spatial Estimation." Proceedings of the IEEE Conference on Computer Vision and Pattern Recognition Workshops. 2018.

[25] Kang, Do-Hun, et al. "Room temperature control and fire alarm/suppression IoT service using MQTT on AWS." 2017 International Conference on Platform Technology and Service (PlatCon). IEEE, 2017.

[26] Mishra, Biswajeeban, and Attila Kertesz. "The Use of MQTT in M2M and IoT Systems: A Survey." IEEE Access (2020).

[27] Krishna, P. Gopi, et al. "Implementation of MQTT protocol on low resourced embedded network." Int. J. Pure Appl. Math. IJPAM 116 (2017): 161-166. 
[28] Mathur, Shubham, et al. "Human detector and counter using raspberry Pi microcontroller." 2017 Innovations in Power and Advanced Computing Technologies (i-PACT). IEEE, 2017.

[29] Jin, Chenghao, Bing Chen, and Feng Hu. "A Drone Formation Transformation Approach." International Conference on Machine Learning and Intelligent Communications. Springer, Cham, 2019.
[30] Barbeau, Michel, Joaquin Garcia-Alfaro, and Evangelos Kranakis. "Geocaching-inspired resilient path planning for drone swarms." IEEE INFOCOM 2019-IEEE Conference on Computer Communications Workshops (INFOCOM WKSHPS). IEEE, 2019.

[31] Ablfazli, Saeid, et al. "Throughput measurement in 4G wireless data networks: Performance evaluation and validation." Computer Applications \& Industrial Electronics (ISCAIE), 2015 IEEE Symposium on. 2015. 